\title{
Guerra trans-especie: Animales en conflicto humano
}

\author{
Joaquín David Rodríguez Álvarez \\ Profesor Asociado, Departamento de Ciencias Histórico-Jurídicas. \\ Facultad de Derecho. Universitat Autònoma de Barcelona
}

Recepción: Julio 2020

Aceptación: Septiembre 2020

Cita recomendada. Rodríguez Álvarez, J.D., Guerra trans-especie: Animales en conflicto humano, dA. Derecho Animal (Forum of Animal Law Studies) 11/3 (2020). - DOI https://doi.org/10.5565/rev/da.509

Resumen

A través del presente artículo se pretende realizar una aproximación desde el anarquismo-verde, como marco teórico, a la utilización/explotación de animales en conflicto bélico, así como al proceso que desemboca en una militarización de los mismos. Mostrando la guerra como un fenómeno inter/intraespecies, donde los anímales han sido y son hoy en día utilizados como armas y medios para conseguir objetivos de tipo de militar/terrorista. Una realidad, que pese a ser sostenida en el tiempo, encontrando ejemplos de todas las épocas y contextos culturales, apenas ha sido objeto de regulación o debate internacional. Generando así, unos vacíos legales que permiten la cristalización de dinámicas de explotación animal que van más allá de los usos abusivos de la industria. Un vacío legal, que, además, condena a los animales a ser parte activa de las dinámicas de conflictividad humanas. El presente artículo aboga en última instancia por la elaboración de mecanismos legales vinculantes a nivel internacional que prohíban el uso de animales en la guerra y garantice la protección de los mismos como víctimas colaterales del conflicto.

Palabras clave: guerra; animales; armamento; combatientes.

\section{Abstract - Trans-species warfare: Animals in human conflict}

This article intends to provide a green anarchism approach as a theoretical framework for the use/exploitation of animals in warfare, as well as the process that leads to their militarization, showing war as an inter/intraspecies phenomenon in which animals have been and are today used as weapons and means to achieve military / terroristic objectives. This is a reality that, despite being sustained over time with examples to be found in all eras and cultural contexts, has hardly been the object of international regulation or debate, generating legal loopholes that allow the crystallization of animal exploitation dynamics that go beyond the abusive uses of the industry, and, furthermore, legal loopholes that condemn animals to be an active part of human conflict dynamics. Ultimately, this article advocates the development of legally binding mechanisms at international level that prohibit the use of animals in war and guarantee their protection as collateral victims of the conflict.

Keywords: war; animals; weapons; combatants 


\section{Introducción}

Their blood stained the land, as they served with pride. A duty to their master, they stood side by side. From the sky, to the sea, through a vast open land, together they fought - both animal and man. (...)

Are they lost in life's story, can we still hear their song? The theatre of war-hell for animal and man whatever the cost, they cannot understand. (Santina Lizzio)

Afirmaba George Santayana que "Solo los muertos han visto el final de la Guerra", una reflexión sobre la pervivencia del conflicto bélico en el mundo, y la normalización del mismo, que sigue estando completamente vigente en la actualidad. Pudiendo así comprender nuestra historia, como una sucesión de enfrentamientos cuyos desenlaces han dado forma el mundo actual, así como a las dinámicas de opresión/subyugación que operan en el mismo.

Podríamos decir así, que somos el resultado de incontables crímenes, perpetrados contra nosotros mismos (como especie) así como contra el resto de especies, muchas de las cuales, han sido forzadas, incluso a batallar por nosotros y nuestros intereses.

Si bien, y pese a la prevalencia del conflicto armado en nuestras sociedades, hemos ido dado pasos como especie hacia la construcción y reconocimiento de marcos teóricos, y legales (vinculantes a nivel internacional), de protección de la dignidad humana, tal y como reflejan en espíritu y letra los Derechos Humanos (en adelante DDHH) así como el derecho humanitario Internacional (DHI), incluso durante el conflicto, tal y como acreditan las Convenciones de Ginebra (CG). Pero apenas hemos comenzado el trayecto que nos conduzca a un reconocimiento de la dignidad de los "otros" habitantes del planeta. Y la urgencia es grande, ya que las guerras no cesan, de la misma manera que no cesan sus consecuencias ecológicas (que parecen acrecentarse exponencialmente con cada conflicto), así como tampoco decrecen las dinámicas de opresión ejercidas sobre otras especies en el marco de conflicto armado.

Un conflicto, que pese a las metamorfosis que ha vivido a través de la historia, continua, en esencia, representando un "modus operandi" de las élites para garantizar la consecución de unos objetivos, internos o externos, que en numerosas ocasiones van más allá del mero interés material (recursos y/o territorio) donde la eliminación física del adversario ha llegado a constituirse como fin y símbolo además de medio, incluso ritual $^{2}$ de autorrealización de una comunidad (tal y como ocurrió con la ejecución extrajudicial de Osama Bin Laden). Y donde el medio natural, ha sido y es considerado, como un simple recurso más, puesto al servicio de la maquinaria de guerra.

Nos encontramos así, ante una realidad, la del conflicto bélico, que hoy en día podemos afirmar que precede, incluso, al propio fenómeno civilizatorio en sí mismo, tal y como afirma Keeley en su obra "War before civilization" 3 . Obra en la que el autor desmonta el consenso académico gestado a lo largo del último medio siglo en el que se consideraba que la guerra prehistórica era rara, inofensiva, y sin importancia, erosionando así el mito del "buen salvaje" y la cosmovisión de carácter primitivista sobre la propia naturaleza de nuestra especie y el significado de la condición humana.

Además, la guerra, parece ser más que un proceso y constructo eminentemente humano ${ }^{4}$ que responde a una dinámicas inseridas en nuestro corpus cultural como escatología material e ideológica cuyos fines, en numerosas ocasiones trascienden a los de la propia estrategia militar ${ }^{5}$. Pudiendo ser también comprendida como una representación material de las pulsiones de muerte freudianas. Pero lo que es más importante, no es

\footnotetext{
${ }^{1}$ SANTAYANA, G., Soliloquies in England and lates soliloquies (Nueva York1933).

${ }^{2}$ BAUDRILLARD, J., The Gulf War did not take place (Indiana 1995).

${ }^{3}$ KEELEY, L. H., War before civilization (Boston 1996).

${ }^{4}$ BAUDRILLARD, J., The Gulf War did not take place (op. cit.).

${ }^{5}$ CHOMSKY, N. La quinta libertad: la intervención de los Estados Unidos en América Central y la lucha por la paz (Barcelona 1999, 1a ed.); ID., On Western Terrorism: From Hiroshima to drone warfare (London 2015). Recuperado de http://ebookespacio.info/onwestern-terrorism-from-hiroshima-to-drone-warfare-pdf-es-descargar-noam-chomsky.pdf (Accedido 14/05/2020). 
un fenómeno exclusivo de nuestra especie, así como no lo son sus afectaciones.

En primer, podemos afirmar que no se trata de un fenómeno exclusivamente humano, porque existen numerosas evidencias sobre la existencia de conflictos "bélicos" entre subgrupos de una misma especie, tal y como muestran las notas de Jane Godall sobre sus observaciones sobre los primates -chimpancés- ${ }^{6}$. Así como las recientes investigaciones realizadas por Joseph Feldblum sobre las mismas ${ }^{7}$ y que se estructuran alrededor de los eventos acaecidos entre 1971 y 1974 en Gombe tras la división de un grupo de chimpancés.

Unos, eventos, que ha sido calificados por algunos autores como "Genocidio pre-humano" . Fundamentalmente debido a los episodios de violencia extrema, orientados a la exterminación del grupo del que los primates se habían escindido, donde determinados hechos tendrían, además, tal y como ocurre en nuestra especie, un contenido simbólico relativo a la construcción de las relaciones de dominio. Claro indicativo de que la guerra (intra-especie) no es un fenómeno exclusivamente humano, aunque ninguna otra especie lo haya llevado a los límites de la nuestra, generando tipologías de armamento que ponen en riesgo no solo a los no-combatientes sino la propia supervivencia de la especie así como de los ecosistemas que habitamos ${ }^{9}$ tal y como sucede con el armamento nuclear.

En segundo lugar, y más allá de eventos generados por la acción de otras especies, la guerra trasciende lo "humano" debido a la victimología, siempre ignorada de los animales durante los conflictos humanos. Tal y como afirma John Loretz:

"A pesar de que cientos de miles de animales han muerto como consecuencia de la guerra humana, no se ha hecho ningún esfuerzo exhaustivo, que yo sepa, para evaluar el número o tipo de víctimas animales durante o después de conflictos pasados. La posibilidad de matar o herir animales nunca ha tenido un efecto disuasorio para quienes toman decisiones sobre la guerra. Algunos acuerdos internacionales recientes, alcanzados para mitigar el impacto de la guerra en el medio ambiente, no se han traducido en restricciones significativas a la actividad militar, y mucho menos en medidas explícitas para proteger a los animales en tiempo de guerra." 10

En este sentido cabe destacar que hoy en día disponemos de algunos estudios de gran interés, aunque escasos, surgidos fundamentalmente a partir de la Guerra de Vietnam, realizados por organizaciones del ámbito del desarme, tal y como la obra "Ecological Consequences of the Second Indochina War" marcado un antes y un después en lo relativo al reconocimiento de los impactos ecológicos de la guerra.

Debemos recordar a este respecto, que de la misma forma que la Segunda Guerra Mundial generó nuevos conceptos (también jurídicos) como el de genocidio o crímenes contra la humanidad, la guerra de Vietnam, nos dejó también una nueva palabra 'ecocidio'. Como consecuencia de los graves impacto climáticos de la misma. Si bien, esta, no fue de ninguna manera la primera guerra en la que se produjo una alteración ecológica. Pero se destaca en la historia moderna como una guerra en la que las acciones internacionales contra el medio ambiente fueron un componente importante de la estrategia y las tácticas de uno de los adversarios -EE.UU- (íbidem). Desarrollando un nuevo tipo de Guerra con afectaciones profundas sobre el clima -Weather-war- ${ }^{12}$ que llevó a cabo una destrucción sistemática del entorno como parte de la estrategia militar, hipotecando el futuro de la región en formas y consecuencias que perduran hasta el día de hoy ${ }^{13} \mathrm{y}$ perdurarán aún más en el tiempo. Una estrategia que hoy en día podemos afirmar que fue mucho más allá en sus efectos que otras estrategias de "tierra-quemada" empleadas por los combatientes en las dos Guerras Mundiales, o a la invasión napoleónica de Rusia, donde las tropas zaristas destruyeron amplias áreas de cultivo, así como el propio ganado con la esperanza de ralentizar el avance francés.

\footnotetext{
${ }^{6}$ GOODALL, J., In the shadow of man (Nueva York 2000).

${ }^{7}$ FELDBLUM, J. T., MANFREDI, S., GILBY, I. C., PUSEY, A. E., The timing and causes of a unique chimpanzee community fission preceding Gombe's "Four-Year War." American Journal of Physical Anthropology 166/3 (2018) 730-744. https://doi.org/10.1002/ajpa.23462

${ }^{8}$ LUND, M. H., Prehuman genocide. Human Evolution, 10/3 (1995) 225-231. https://doi.org/10.1007/BF02438975. Ver SALAS, J., El cruel asesinato que desató la primera guerra entre primates no humanos de la historia | Materia (14/01/2014). Recuperado de $\mathrm{http}: / /$ esmateria.com/2014/01/14/el-cruel-asesinato-que-desato-la-primera-guerra-entre-primates-no-humanos-de-la-historia/ (Accedido 18/04/2020).

${ }^{9}$ FETTER, S., Ballistic missiles and weapons of mass destruction: What is the threat? What should be done? International Security Journal 1/16 (1991) 5-42.

${ }^{10}$ LORETZ, J., The animal victims of the Gulf War. Phisicians for Social Responsability (1991) 221-225. Recuperado de https://www.ippnw.org/ ippnwor1/pdf/mgs/psr-1-4-loretz.pdf (Accedido 14/05/2020).

${ }^{11}$ SIPRI., Ecological Consequences of the Second Indochina War (1976).

12 DARACK, E., Weaponizing Weather: The Top Secret History of Weather Modification. Weatherwise 72/2 (2019) 24-31. https://doi.org/10.1080/00431672.2019.1559400

13 KLEM, E., BAKRAČ, S., MILANOVIĆ, M., Ecological consequences of warfare. Vojno Delo, 69/4 (2017) 126-133. https://doi.org/10.5937/vojdelo1704126K
} 
En la actualidad, también contamos con algunas informaciones y estudios relativos a los daños ocasionados por tropas combatientes sobre el ganado, a este respecto Lorenz indica que, en la primera guerra del Golfo, el $80 \%$ por del ganado kuwaití fue aniquilado ${ }^{14}$, lo que nos da una idea de los daños que el conflicto humano genera sobre otras especies.

Además, y aparte del ganado y de los anímales que habitan de forma endógena en el área de conflicto, existe otra tipología de víctima animal en conflicto humano que debe ser tenida en cuenta, por ser generalmente olvidada. Tal y como son los animales confinados en zoos, que en ocasiones pueden verse transformados en zona de guerra, cuyo ejemplo paradigmático puede ser el de Sarajevo, y la triste masacre de animales que tuvo lugar en el mismo, en $1992^{15}$ cuando todos los animales, excepto un oso, perecieron a causa del asedio serbio sobre la ciudad. No es extraño que los animales confinados en los zoos, mueran a causa de los ataques, se conviertan en sustento de las tropas o civiles, o sea incluso utilizados como medio para generar el caos entre los locales. Animales que son siempre concebidos como medio, bajo una imperante aproximación antropocéntrica, no solo al conflicto sino a la jerarquía de relaciones inter-especie. Siendo esta precisamente una de las claves del anarquismo-verde como marco teórico, debido a su intento, a partir de las tesis ecologistas libertarias, de establecer una crítica más allá de las interacciones humanas, incluyendo las interacciones entre humanos y no humanos en base a nociones de poder/sometimiento.

Y finalmente, y, en tercer lugar, el conflicto bélico humano, trasciende los límites de nuestra especie debido a lo que sería la propia utilización de animales como parte inherente del conflicto, objeto central del presente artículo. Ya sean estos usos como medio logístico (transporte de munición, maquinaria bélica, recursos a las líneas del frente) rescate, así como armamento en sí mismo. Una tendencia que si bien es cierto, lleva siglos produciéndose, en el tiempo actual ha experimentado un crecimiento exponencial (en lo relativo al número de especies involucradas), debido fundamentalmente al vacío legal existente en los marcos jurídicos que regulan la Guerra -ius in bello- ${ }^{16}$. Así como gracias a la mentalidad humanista imperante, que tal y como veremos más adelante, continúan imbuyendo a los animales de una conceptualización meramente patrimonialista. Si bien, esta tendencia parece estar evolucionando en la actualidad, encontrando intentos destinados a otorgar la consideración de combatiente a los animales participantes activos de conflictos armados, pese a que dicha tendencia, solo representaría una cristalización de las dinámicas de opresión interespecie, imbuidas por el mito del "héroe animal" combatiente. Un mito que operaría con un parámetro similar a los del "héroe-soldado" humano. Como método de justificación de la participación en el conflicto, propio de los parámetros de las masculinidades militarizadas.

\section{2. ¿Animales combatientes?}

La participación de animales de forma activa dentro de los marcos del conflicto armado va intrínsecamente ligado al propio proceso de subordinación del resto de especies a "lo humano", estrechamente vinculado a los procesos de domesticación -cosificación/reificación- y normalizado dentro del imaginario colectivo, cuando no magnificado y mitificado. ¿Quién no ha oído hablar del poderoso contingente de elefantes de Anibal Barca y su travesía de los Alpes? Aunque solo fueran 38, la mayoría de los cuales perecieron en las montañas, y en las marismas etruscas, sirviendo el único superviviente como montura del propia Aníbal ${ }^{17}$. Elefantes, caballos, camellos, perros, palomas y un largo etcétera de especies subordinados y sometidos a un instinto de muerte demasiado humano ${ }^{18}$ utilizados como medio y nunca comprendidos como fin en sí mismo ${ }^{19}$.

Una larga historia de conflicto y uso animal que muchas veces se ha traducido en reconocimientos de tipo simbólico alimentando unas meta-narrativas completamente nocivas de "heroicidad animal" en tiempos de Guerra que se manifiesta a través de diversas estrategias orientadas a contrarrestar la propia realidad opresiva que determina dichas relaciones humano-animal sobre el campo de batalla; Como cuando el 21 de Julio de 1994, en el 50 aniversario de la invasión de Guam, se inauguró en el Cementerio de perros del cuerpo

\footnotetext{
${ }^{14}$ LORETZ, J., The animal victims of the Gulf War. Phisicians for Social Responsability (op. cit.).

15 BURNS, J., Sarajevo Journal; In the Zoo's House of Horrors, One Pitiful Bear. NYTimes (16/10/1992). Recuperado de: https://www.nytimes.com/1992/10/16/world/sarajevo-journal-in-the-zoo-s-house-of-horrors-one-pitiful-bear.html (Accedido 14/06/3030); HEDIGER, R., Animals and War: Studies of Europe and North America (Leiden/Boston 2013).

${ }^{16}$ NOWROT, K., Animals at War: The Status of "Animal Soldiers" under International Humanitarian Law on JSTOR. Historical Social Reserach 154/2 (2015) 128-150.

${ }^{17}$ MACGREGOR, M., The story of Rome (Londres 2006) 38.

${ }^{18}$ FREUD, S., Beyond the Pleasure Principle. The Standard Edition of the Complete Psychological Works of Sigmund Freud, Volume XVIII (1920-1922): Beyond the Pleasure Principle, Group Psychology and Other Works (Londres, 1920) 1-64. https://doi.org/10.3366/pah.2015.0169

${ }^{19}$ KANT, I., Fundamentación para una metafísica de las costumbres (Madrid 2002); SCHOPENHAUER., A., The World as Will and Representation. Philosophy and Phenomenological Research, Vol. 20 (London 1959). https://doi.org/2104368

68 Derecho Animal. Forum of Animal Law Studies, vol. 11/3
} 
de Marines de los Estados Unidos el memorial dedicado a los 25 perros asesinados durante la "liberación de Guam en 1994, o cuando el 24 de Noviembre de 2004 se inauguró en Londres el Memorial de Guerra de los Animales "fallecidos" en combate ${ }^{20}$.

Unos reconocimientos de tipo simbólico que han ido acompañados sistemáticamente de un vacío legal tanto a nivel Internacional como nacional, existiendo apenas un puñado de ejemplos normativos, que han tenido como objeto el reconociendo de los animales como sujetos pasivos de derechos. Así el Congreso de los Estados Unidos adoptó una ley en diciembre de 2012 - Canine Members of the Armed Forces Act ${ }^{21}$ dirigida a la protección y el apoyo de los perros militares retirados. Una norma que, entre otras cosas, tenía por objetivo racionalizar el proceso de adopción, además de autorizar la creación de un sistema de atención veterinaria para estos caninos. Cabe desatacar que antes de la aprobación de dicha norma una gran parte de dichos animales eran asesinados una vez finalizada su "vida útil" como ejemplo paradigmático de los extremos a los que hemos llegado en el proceso de cosificación y subordinación de otras especies.

También, han existido y existen en la actualidad otro tipo de prácticas, que afectan particularmente a los perros, a quienes se les da ocasionalmente, $\mathrm{y}$ al menos no oficialmente, rangos militares más altos "que los hacen superiores a sus manejadores, una práctica diseñada para garantizar que los humanos traten a los animales con deferencia. Tienen un parche de rango en su armadura" ${ }^{22}$. Pero que rara vez ha afectado a otro tipo de especies involucradas en el conflicto (ha habido casos de algunos caballos). Aunque si han servido para nutrir el imaginario colectivo de símbolos y mitos sobre la participación de los animales en conflicto. Actuando no solo como justificación, sino como normalización de dicha relación bélica.

Es más, los marcos jurídicos legalmente vinculantes del -ius in Bello- ignoran sistemáticamente el rol de los animales, existiendo a penas algunas referencias relativas a los impactos ecológicos de la Guerra, si bien siempre bajo marcos mentales completamente antropocéntricos ${ }^{23}$ tal y como ilustra el requisito adicional consagrado en el artículo 55 (1) del Protocolo de la CG de 1977 que estipula que el daño al medio ambiente solo es relevante para los propósitos de esta disposición si está destinado o puede esperarse que también "perjudique la salud o la supervivencia de la población".

Un enfoque que puede observarse, a su vez, presente en la siguiente declaración hecha por la Corte Internacional de Justicia en su opinión consultiva sobre la legalidad de la amenaza o el uso de armas nucleares del 8 de julio de 1996: "La Corte también reconoce que el medio ambiente no es una abstracción sino que representa el espacio vital, la calidad de la vida y la salud misma de los seres humanos, incluidas las generaciones por nacer" ${ }^{24}$ donde una vez más se comprende a los animales, como un mero complemento al desarrollo y sostenibilidad de nuestra especie, carente de hecho de cualquier derecho. Tal y como reconoce Nowrot $^{25}$. Es decir, el medio se concibe como un recurso que debe ser protegido en tanto y cuanto es necesario para nuestro desarrollo, pero no algo que deba ser protegido "per se".

El problema, reside por tanto en la propia concepción ético-filosófica que relaciona nuestra existencia en el planeta con una serie de medios de subsistencia necesarios para nuestra supervivencia, que ha retroalimentado la configuración de un -ius in Bello- que pese a reconocer el impacto que el ejercicio bélico tiene sobre los ecosistemas (un impacto que es completamente abrasivo con el entorno), este solo es relevante si condiciona el futuro desarrollo humano tanto desde una perspectiva intergeneracional como intrageneracional. Cristalizando así una jerarquización antropocéntrica, que cosifica y subordina de manera clara y abrumadora al resto de especies al interés humano.

Si bien cabe destacar, diversas tendencias normalizadoras, que han surgido en los últimos años dentro del espectro del derecho animalista, que han realizado llamamientos a la configuración de marcos legales que regulen la participación de animales en el conflicto a partir del reconocimiento de los mismo como sujetos activos de derecho. A este respecto cabe destacar la aportación de Nowrot $^{26}$ en lo que podría considerarse como una ampliación de un incipiente derecho ambiental internacional ${ }^{27}$. Con aportaciones destacables de

\footnotetext{
${ }^{20}$ KEAN, H., Animals and war memorials: Different approaches to commemorating the human-animal relationship. In Animals and War (2013) 237-262. Brill. Recuperado de https://brill.com/view/book/edcoll/9789004241749/B9789004241749-s012.xml (Accedido 14/06/2020).

${ }^{21}$ BLUMENTHAL, R. S. 2134 - 112th Congress (Washington 2011-2012): Canine Members of the Armed Forces Act (2012).

${ }^{22}$ LONDONO, E., Military Dog Captured by Taliban Fighters. Washington Post (28/04/2014). Recuperado de https://www.washingtonpost.com/world/national-security/military-dog-captured-by-taliban-fighters-who-post-video-of-theircaptive/2014/02/06/c8d0f8f0-8f44-11e3-84e1-27626c5ef5fb_story.html (Accedido 15/06/2020).

${ }^{23}$ RODRÍGUEZ-ÁLVAREZ, J., Rethinking Paradigms in the Technolo-ecological Transition. Journal of Law 1/2 (2019) 169-184.

${ }^{24}$ ICJ., Legality of the Threat or Use of Nuclear Weapons, Advisory Opinion (1996). ICJ-Reports.

${ }^{25}$ NOWROT, K., Animals at War: The Status of “Animal Soldiers” under International Humanitarian Law (op. cit.) 135.

${ }^{26}$ Ibíd.

${ }^{27}$ BODANSKY, D., The art and craft of international environmental law (2010). Recuperado de https://books.google.es/books?hl=es\&lr=\&id=eVeuV1EGna8C\&oi=fnd\&pg=PR7\&dq=international+environmental+law\&ots=8EtB bmCzlc\&sig=WFkDTHPuHl18bfTkzJoifamaEgQ (Accedido 14/06/2020); SANDS, P., Principles of International Environmental Law (2012). Recuperado de
} 
elementos orientadas a reconocer a los animales como sujetos combatientes, en parte gracias a la ambigüedad de la conocida como cláusula Marteens de las Convenciones de Ginebra ${ }^{28}$.

Pero este reconocimiento de los animales como combatientes, representa serios problemas teóricos, en tanto y cuanto combatiente es el estado legal de un sujeto que tiene derecho a participar en hostilidades durante un conflicto armado internacional. La definición legal del cual se encuentra en el artículo 43 (2) del Protocolo Adicional Uno (AP1) a los Convenios de Ginebra de 1949. Establece que "los miembros de las fuerzas armadas de una Parte en conflicto (que no sea personal médico y los capellanes cubiertos por el artículo 33 de la Tercera Convención) son combatientes, es decir, tienen derecho a participar directamente en las hostilidades".

Además de tener derecho a participar en las hostilidades, los combatientes tienen derecho al estado de Prisioneros de guerra cuando son capturados durante un conflicto armado internacional ${ }^{29}$. "Si bien todos los combatientes están obligados a cumplir con las normas de derecho internacional aplicables en los conflictos armados, las violaciones de estas normas no privarán a un combatiente de su derecho a ser combatiente o, si cae en el poder de una Parte adversa, de su derecho a ser prisionero de guerra" ${ }^{30}$. Tal y como ocurrió con un perro de guerra británico capturado por los Talibanes durante la invasión de Afganistán ${ }^{31}$.

Si bien, esta teórica consideración del animal combatiente alberga una serie de problemas el primero de los cuales se deriva precisamente, del voluntarismo de la acción de participación, o consentimiento. Problema que experimentan otro tipo de combatientes (no reconocidos legalmente como tales) cuya propia naturaleza relativa al conflicto infiere problemas de categorización tal y como puede ser el rol de los niños soldado.

En este sentido cabe recordar que el derecho internacional humanitario (DIH) prohíbe el reclutamiento y el uso de niños en hostilidades. Una prohibición que es aplicable tanto en conflictos armados internacionales como no internacionales. Así, estas prohibiciones sobre el reclutamiento de niños menores de 15 años están consagradas en el Artículo 77 del Protocolo Adicional I, y en el Artículo 4 del Protocolo Adicional II también se consideran prohibiendo la aceptación del alistamiento voluntario (PI, Art. 77 (2); P II, Art. 4 (3) (c)).

Una prohibición que se estructura fundamentalmente a partir, no de un concepto de voluntarismo, es decir, si el niño en cuestión ha sido forzado o no a participar en el conflicto sino en base a su -mayor susceptibilidad a la influencia- es decir, la falta de plena conciencia alrededor de la acción ejercida y la respectiva responsabilidad derivada. Hecho que no invalida en ningún momento que el niño sea considerado un agente moral, sino que entiende, que el ejercicio de dicha agencia se produce a través de intersubjetivación a través de un tercero ${ }^{32}$.

Y es que la cuestión de la consideración de agente moral, resulta crucial a la hora de interpretar un individuo como sujeto activo o pasivo de derechos (sobre todo respecto a los usos de la guerra en el binomio combatiente, no combatiente), si bien, resultaría un error pensar que esta agencia es una categoría exclusiva de los humanos ${ }^{33}$.

Ya que, si bien es cierto, que numerosos filósofos han argumentado que la agencia moral es característica exclusiva de nuestra especie y que su ausencia de otros animales justifica otorgar un mayor estatus moral a los humanos. Recientes estudios parecen demostrar lo contrario, substrayendo de esta forma a los humanos el monopolio de la agencia moral, que se admite en diversos grados para otras especies y no requiere el dominio de los principios morales. La opinión de que todos y solo los humanos poseemos agencia moral indicaría simplemente nuestra subestimación de la vida mental de otros animales. Dado que muchos otros animales son agentes morales (en diversos grados), y también están sujetos a obligaciones morales (limitadas) $^{34}$.

Es decir, un animal es susceptible de ser considerado agente moral, si bien y en lo relativo a su

https://books.google.es/books?hl=es\&lr=\&id=Cz8hAwAAQBAJ\&oi=fnd\&pg=PR5\&dq=international+environmental+law\&ots=ef BBbwBntt\&sig=N5MO47bxGX69hwZfX4HRXrJ5Y8Y (Accedido 04/06/2020).

${ }^{28}$ MERO, T., The Martens Clause, principles of humanity, and dictates of public conscience. American Journal of International Law 94/1 (2000) 78-89. Recuperado de https://www.cambridge.org/core/journals/american-journal-of-international-law/article/martensclause-principles-of-humanity-and-dictates-of-public-conscience/F55EECE5BED3DDB9D78162DA4509A03A $11 / 05 / 2020)$.

${ }^{29}$ Tercera Convención de Ginebra. Artículo 4 (A) (1)

${ }^{30}$ Protocolo adicional 1 (Convenciones de Ginebra). Articulo 44 (2)

${ }^{31}$ BBC., Afghan Taliban capture British military dog - BBC News (Londres 06/02/2014). Recuperado de https://www.bbc.com/news/world-asia-26062679 (Accedido 24/06/2020); LONDONO, E., Military Dog Captured by Taliban Fighters. Washington Post (op. cit.).

${ }^{3}$ SÁNCHEZ-VẢZQUEZ, M. J., Ética e infancia: el niño como sujeto moral. Fundamentos En Humanidades 15/1 (2007) $179-193$.

${ }^{33}$ CARTER, B., CHARLES, N., Animals, Agency and Resistance. Journal for the Theory of Social Behaviour 43/3 (2013) 322-340. https://doi.org/10.1111/jtsb.12019

${ }^{34}$ SHAPIRO, P., Moral agency in other animals. Theoretical Medicine and Bioethics 27/4 (2006) 357-373. https://doi.org/10.1007/s11017-006-9010-0 
participación en un conflicto humano, esta incipiente moralidad se vería intersubjetivada debido a la lealtad hacia el "cuidador", siendo incapaz de comprender la magnitud del conflicto, o la propia naturaleza éticomoral del mismo.

Lo que, estructurado de otra forma, vendría a significar que la participación del animal en el conflicto deriva de un régimen de subyugación/domesticación donde el interés humano se constituye como eje articulador de las relaciones intra-especie y donde el animal es simplemente un objeto de guerra y no un sujeto en la misma. Siendo por tanto imprescindible redefinir el propio contexto de la relación en base a una noción que supere los marcos básicos del antropocentrismo ${ }^{35}$, considerando a los animales como iguales en un sentido ecológico del termino en lo relativo a las aportaciones de cada especie para la propia supervivencia y desarrollo del ecosistema.

Si bien, antes de abordar los marcos teóricos que pueden albergar una nueva comprensión de los animales durante el conflicto, es necesario abordar sus usos a fin comprender la multiplicidad de escenarios en que los animales son sometidos con finalidades bélicas, y como su conceptualización se aproxima más a la de arma, que la de combatiente. Siendo por tanto dicha consideración última, un simple ejercicio de cristalización de las dinámicas de opresión existentes en el mundo actual, y en nuestra relación histórica con el resto de especies que habitan el planeta.

\subsection{Animales como herramienta y arma}

Resulta extremadamente complejo establecer un índice claro de especies utilizadas en conflicto armado, así como de los programas que se desarrollan en la actualidad para estos fines, debido, principalmente, a la opacidad con la que operan la mayor parte de los programas de defensa. Siendo incluso, mucho más complejo establecer los criterios éticos de entrenamiento a los que son sometidos los animales. Si bien, a través del presente epígrafe se pretende desmitificar el rol del animal como "combatiente" en pos de su comprensión como "arma", es decir objetivizado y subyugado al interés humano a lo largo de nuestra historia bélica, en usos que han vulnerado la dignidad intrínseca de los mismos como traslación de unas dinámicas de explotación propiamente humanas y cuyo final todavía no somos capaces de atisbar.

El uso de animales en el marco del conflicto, tal y como hemos ido mostrando, es tan antiguo como el propio proceso de domesticación, momento en el que podríamos decir que dejamos de estar en "guerra" con el medio, para empezar su dominio y subyugación. Y si bien es cierto que este uso en nuestro tiempo, no es tan extensivo en lo relativo a el número absoluto de animales implicados en el conflicto, sobre todo si lo comparamos con las épocas de uso intensivo de la caballería, sí que es mayor en lo referente al número de especies involucradas en el mismo. Así como en lo relativo a líneas de investigación abiertas para la militarización y entrenamiento de otras especies.

Así, hoy en día, continuamos empleando especies, que ya se han utilizado en la antigüedad, y que, a día de hoy, podríamos decir siguen en "activo", tal sería el ejemplo de las abejas, cuyo uso militar está bien documentado desde época romana, como en el caso del asedio de Themiscyra, llevado a cabo por Lucullus en el año 72 a.c:

"Los sitiadores de este lugar levantaron torres, construyeron montículos y cavaron túneles tan grandes que se podían librar batallas subterráneas en ellos. Los habitantes cortaron aberturas en estos túneles desde arriba y empujaron a los osos y otros animales salvajes y enjambres de abejas contra los trabajadores." 36

O como explica Bishop en su obra "Robbing the bees": "Para las batallas navales, los romanos desarrollaron catapultas especiales de enjambre a bordo. Criaban abejas y las mantenían en colmenas de tierra livianas y frágiles con el único propósito de lanzarlas a los barcos enemigos. Las abejas enojadas desconcertarían tanto a los marineros opuestos que a menudo saltaban por la borda para escapar." ${ }^{37}$. Usos que nunca han cesado, y parecen acreditados por otras fuerzas recientes, como el Vietcong, entre otros ${ }^{38}$.

Si bien, además de estas especies (abejas, caballos, camellos, perros, elefantes...) que han tiene una larga trayectoria de uso militar, existen nuevas investigaciones sobre otras especies y sus potenciales usos bélicos.

\footnotetext{
${ }^{35}$ RODRÍGUEZ-ÁLVAREZ, J., Rethinking Paradigms in the Technolo-ecological Transition (op. cit.).

${ }^{36}$ WHITE, H. (n.d). Mithridatic Wars. 11.78. Recuperado de

http://www.perseus.tufts.edu/hopper/text?doc=Perseus\%3Atext\%3A1999.01.0230\%3Atext\%3DMith. (Accedido 04/06/2020)

${ }^{37}$ BISHOP, H., Robbing the bees (Nueva York 2006).

${ }^{38}$ DELANEY, L., Military apllications of apiculture: The (other) nature of war (2000). Recuperado de https://apps.dtic.mil/dtic/tr/fulltext/u2/a600636.pdf (Accedido 03/04/2020).
} 
Bob Bailey, el primer director de entrenamiento para el programa pionero de delfines de la Marina "Navy's pioneering dolphin program", quien a lo largo de sus años de servicio, ha trabajado con un amplio abanico de especies, desde enseñar a los delfines a detectar submarinos hasta inventar el Bird Brain, un aparato que permitía a una persona jugar tick-tack-toe contra un pollo afirmaba en una entrevista a National Geographic "Nunca encontramos un animal que no pudiéramos entrenar" ${ }^{39}$.

Si bien, a la hora de comprender el porqué de la obsesión que han mantenido los ejércitos del todo el mundo en investigar sobre usos de animales en conflicto bélico, debemos tener en cuenta, que en algunas ocasiones, el uso de los animales como arma, ha transformado el curso de la historia. Así, el historiador Lynn White ${ }^{40}$ en su obra "Tecnología Medieval y cambio social" habla sobre la importancia de un artefacto que en principio puede resultar bastante sencillo e insignificante a nuestros ojos tal y como es el estribo, pero clave en uno de los procesos sociológicos más importantes de la historia de Europa ${ }^{41}$. Según White, la introducción y difusión del estribo en la sociedad europea fue una de las principales causas de la aparición de la sociedad feudal entendida como una sociedad dominada por la aristocracia guerrera y propietaria de la tierra.

El estribo hizo posible una nueva unidad de combate, una máquina de guerra sin precedentes: la combinación de un hombre, una espada y un caballo. El estribo permitía el uso de la espada con mucha estabilidad y sin el peligro, antes permanente, de caerse del caballo cada vez que el golpe no acertaba al enemigo. La aparición de esta nueva unidad de combate, el caballero, exigía sin embargo un entrenamiento sistemático, además de caballos especiales y de armaduras diseñadas específicamente para defenderse de otros caballeros. Estas condiciones específicas hicieron necesaria la creación de una organización social que pudiera garantizar el mantenimiento de esta nueva elite ociosa ${ }^{42}$ de guerreros a caballo: esta nueva organización es la que conocemos con el nombre de feudalismo. Claro ejemplo de los impactos que la militarización de otras especies ha tenido sobre el desarrollo de la nuestra.

Si bien, no es necesario regresar a la Edad Media para buscar ejemplos de usos de animales de forma regular en conflicto bélico, ya que hoy en día existen ejemplos de sobra conocidos sobre unidades animales estables que forman parte de fuerzas armadas regulares, como la Unidad Oketz de la Fuerzas de Defensa de Israel (IDF). Esta Unidad "opera con perros especialmente entrenados para misiones en el campo de la lucha contra el terrorismo, búsqueda y rescate y otros fines específicos" ${ }^{3}$.

Así como ejemplos de animales que han sido utilizados, incluso muertos, como arma. Tal y como muestran documentos desclasificados del SOE (Special Operations Executive) de su catálogo de "Special Devices" utilizados en el contexto de la Segunda Guerra mundial por tropas británicas, como el caso de la "rata explosivo" 44 .

Usos, que cada vez se extienden, tal y como mencionábamos previamente, a un mayor número de especies, tal y como pueden ser los delfines.

Así, en 2014, cuando Rusia ocupó Crimea, una unidad militar ucraniana, encontró varios "delfines de combate". Se cree que los mamíferos marinos se usaban para encontrar objetivos submarinos como minas o para bloquear la entrada de intrusos en áreas restringidas ${ }^{45}$.

De la misma forma, en 2015, Hamas afirmó que, detuvieron a un delfín que estaba espiando para las fuerzas israelíes. El diario The Times of Israel informó de acusaciones de que el delfín estaba equipado con equipo de espionaje, que incluía, entre otros, cámaras ${ }^{46}$.

Un uso de estos mamíferos que ya viene acreditado desde la década de los 60, momento en el que la Marina de los EE. UU. Ejecutó un programa de investigación sobre los usos militares de los mismo. Un representante del programa de investigación de mamíferos marinos de la Universidad de Hawái dijo, en declaraciones a National Geographic en 2014, que: "Estados Unidos no solo ha usado delfines como guardias, sino que los animales también son muy hábiles para detectar minas submarinas. La geolocalización de los delfines es tan precisa que incluso se han utilizado en lugar de máquinas." 47

Abejas, delfines, caballos, perros, camellos, elefantes y un largo etc. de especies que han sido militarizados y convertidos en arma. Subyugados bajo unas dinámicas de explotación inherentes a nuestra

\footnotetext{
${ }^{39}$ VANDERBLIT, T. The CIA's Most Highly-Trained Spies Weren't Even Human (Washington 2013).

${ }^{40}$ WHITE, L., Tecnología medieval y cambio social (Barcelona 1973).

${ }^{41}$ RODRÍGUEZ, J., La civilización ausente: Tecnología y sociedad en la era de la incertidumbre (Oviedo 2016, 1a ed.).

${ }^{42}$ VEBLEN, T., The Theory of the Leisure Class. A Penn State Electronic Classics Series Publication (Oxford 2009)

${ }^{43}$ IDF., OKETZ Unit (2020). Accedido 15/06/2020, de https://www.idf.il/en/minisites/oketz-unit/

44 UK, N. A., World War II (2020). Recuperado de https:/www.nationalarchives.gov.uk/education/worldwar2/theatres-ofwar/western-europe/investigation/resistance/sources/docs/4b/ (13/05/2020)

${ }^{45}$ GIBBENS, S., Why These Animals Were Accused of Being International Spies. National Geographic (Londres 2018). Recuperado de https://www.nationalgeographic.com/news/2018/02/lizard-nuclear-spying-animal-espionage-spd/ (Accedido 12706/2020).

46 Ibídem

${ }^{47}$ Ibídem

72 Derecho Animal. Forum of Animal Law Studies, vol. 11/3
} 
especie $^{48}$. Siendo precisamente, la regeneración de nuestros marcos mentales, el paso previo a la restructuración y desmantelación de dichas dinámicas de opresión inter-especie.

Siendo, además necesario romper la mitificación del "soldado héroe" o el "animal héroe" ya que en las guerras rara vez existen más héroes que los desertores. Debido a que esta meta-narrativa se asienta e infiere precisamente sobre el voluntarismo del animal en su participación y acciones. Obviando el proceso de subyugación que le ha llevado en primer lugar hasta el campo de batalla. Dotando al hecho de un cierto romanticismo, derivado de la construcción de masculinidades militarizadas donde la autorealización del "yo" queda supeditada a la "gesta" o "acto heroico" en servicio. La muerte por la patria que acerca a los patios del Valhalla. Una conceptualización que pese o lo ridícula que suena, sobre todo si se analiza desde una óptica vitalista, sigue imbuyendo no sólo a la épica militar, sino a la sociedad en su conjunto.

\section{Hacia una configuración Post-humana del conflicto y de la seguridad}

Sin lugar a dudas, la reubicación de los animales en el contexto del conflicto humano, pasa por una superación del antropocentrismo, comprendido como la herencia envenenada que la teología cristiana fue capaz de trasladar a las bases conceptuales del humanismo. Basadas en la configuración de un Edén que se articuló a modo de jardín de juegos de nuestra especie, donde la naturaleza no es más que un "regalo de dios" al que se nos invita a usar y disfrutar a nuestro antojo. Donde su mera existencia es representada y concebida como una subalteridad a "lo-humano":

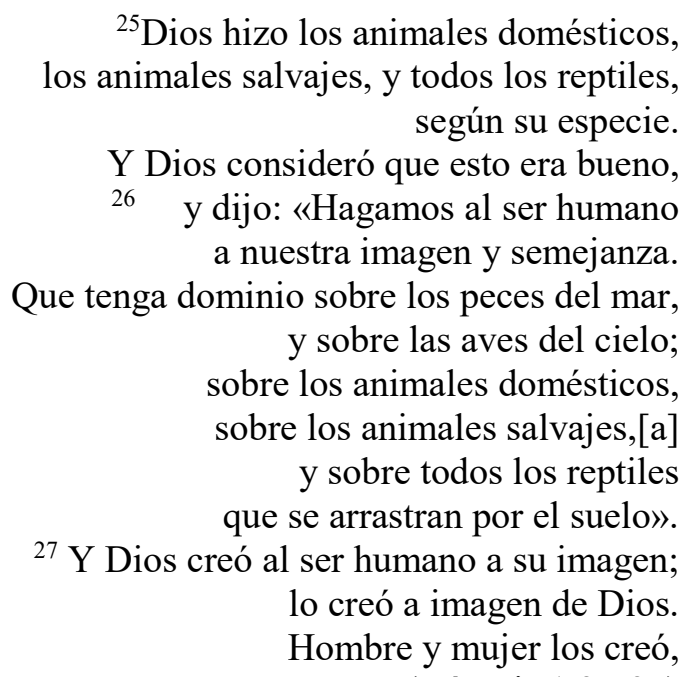

(Génesis 1:25-27)

Generando así un marco conceptual que ha justificado a lo largo de los siglos no solo el dominio, sino cualquier tipo de práctica por abusiva y degradante que fuera, al establecer de facto, una jerarquía interespecies. Jerarquía que a su vez se ve reflejada en los marcos legales contemporáneos, incapaces de reconocer como sujetos activos de derechos al resto de especies que co-habitan el planeta con nosotros, las cuales continúan ocupando en los marcos mentales y legales occidentales, el rol otorgado por la tradición judeo-cristiana. Ignorando así completamente la importancia del resto de especies a fin de mantener unos equilibrios ecológicos, extremadamente erosionados por la acción humana, que resultan claves para sustentar la propia vida sobre el planeta.

Es por ello, imprescindible, avanzar en la construcción de marcos conceptuales post-humanos ${ }^{49}$ que realicen una superación efectiva del antropocentrismo, considerando al resto de especies como no-supeditadas a la nuestra, y por tanto interdependientes a nuestras dinámicas, en base a lo que podría considerarse como una aproximación materialista, tanto desde el punto de vista dialéctico, como de una nueva praxis. Superando la propia conceptualización de "hombre" referido a las ramificaciones de su representación vitrubiana, que ha sido tradicionalmente comprendido como postulado universal pese a que esta imagen arquetípica coincide implícitamente sólo con el varón, blanco, urbanizado, hablante de un idioma estándar, heterosexual inscrito en la unidad reproductiva base, ciudadano de pleno derecho de una comunidad política reconocida ${ }^{50}$. Es decir,

\footnotetext{
${ }^{48}$ BOOKCHIN, M. Ecología y pensamiento revolucionario (Mallorca 2019, 1a ed.).

${ }^{49}$ BRAIDOTI, R., Lo Posthumano (Barcelona 2015, 1a ed.).

${ }^{50}$ DELEUZE, G., GUATTARI, F., Millepiani (Roma 2006).
} 
quizás la imagen menos representativa de la realidad contemporánea ${ }^{51}$. Pero cuyos marcos continúan delimitando y constriñendo cualquier tipo de alteridad o divergencia.

En este sentido, la filosofía que ha sustentado la comprensión de la Guerra, desde los múltiples ángulos existente tal y como es abordada por Moseley en su obra "A philosophy of war" ${ }^{52}$ ha pasado tradicionalmente por alto, cualquier aproximación inter-especies, siendo considerada, no ya como un fenómeno eminentemente humano, sino obviando cualquier tipo de implicación de los animales en la misma. De San Agustín a Marx, cualquier reconocimiento del sufrimiento, uso y abuso de la "otredad" animal queda subyugada, obviada, y deliberadamente ignorada.

Siendo por tanto vital, avanzar en la consecución de un paradigma post-humano, que reorganice nuestras relaciones ontológicas y epistemológicas con el medio y las diferentes especies que lo habitan. Un proceso que debería pasar, sin ninguna duda, por una desestructuración, en primer lugar de las jerarquías, en base a una praxis orientada a la generación de marcos de acción de liberación tal y como proponen numerosos autores vinculados a escuelas de pensamiento anarquistas ${ }^{53}$. Una liberación que por lo que respecta al conflicto debe pasar por restringir la participación en el mismo, a todo sujeto animal o humano, que no pueda ejercer plena autonomía moral en lo relativo a la comprensión del mismo.

Es decir, el camino no se orientaría hacia un reconocimiento del "animal" como combatiente lo que le otorgaría de facto una serie de derechos. Hecho que podría ser considerado como una victoria (parcial) del movimiento ambientalista/animalista, sino por la asunción básica de que los animales no deber ser utilizados en conflictos humanos. Unos conflictos que de facto y a lo largo de la historia han sido conflictos Inter/intra especies en base a unas asunciones de raza, nación y clase que lejos quedan de los marcos instintivos del resto de especies ${ }^{54}$.

Es por ello necesario abogar por una prohibición clara, que podría estructurarse como tratado internacional o como protocolo adicional a la Convención sobre ciertas armas convencionales de Naciones Unidas (1983), que en la actualidad ya cuenta con cinco protocolos adicionales ${ }^{55}$. Una propuesta, está última, que, si bien no acabaría de solucionar el reconocimiento de los animales como sujetos activos de derechos, ya que les sometería a la cosificación de ser transformados, aunque solo sea conceptualmente, en armas. Pero que permitiría restringir sus usos en conflicto humano. Siendo necesario tener en cuenta que históricamente, su conceptualización ha sido más cercana a la de arma que a la de combatiente.

Unos equilibrios legales complejos, dentro de un sistema legal que apenas da márgenes de maniobra en lo que se refiere a la protección de otras especies, a partir de su propia identidad, en los marcos del conflicto humano. Y que sin lugar a dudas debe avanzar hacia la configuración de un Derecho Internacional Animal, que ofrezca marcos de actuación claros, sobre su interdependencia respecto a nuestra especie, y su independencia respecto a nuestros conflictos. Es decir, considerando al resto de especies como sujetos activos de derecho, y no como un mero soporte al desarrollo de nuestra especie. Si bien, este camino es necesario recorrerlo fuera de los marcos del ius in bello, ya que dentro de estos marcos, poco más se puede hacer que aspirar a prohibir la participación de animales en conflicto, y a reducir los impactos ecológicos de la guerra.

\section{Conclusiones}

La subyugación del resto de especies a lo humano, encuentra en la expresión militar la cúspide de su cosificación. Degradando al animal a la categoría de herramienta para la consecución de unos fines a través de la violencia. Es decir, comprendiendo a los animales como parte intrínseca de la maquinaria bélica (alimento, logística, armamento...), o incluso en objetivo en sí mismo en las prácticas de estrategias de "tierraquemada" o "weather-war".

Unos animales que en el marco del conflicto bélico han sido situados, tradicionalmente tal y como hemos podido observar, mucho más cerca de la categorización de arma que de la de combatiente. A pesar de los meta-relatos construidos alrededor del "héroe" canino al que se le erigen monumentos, cementerios y se le otorgan rangos y condecoraciones. Una narrativa que ha sido utilizada de forma reiterativa para dulcificar

\footnotetext{
${ }^{51}$ IRIGARAY, L., Speculum. L'altra donna (Roma 2010); BRAIDOTI, R., Lo Posthumano (op. cit.)

${ }^{52}$ MOSELEY, A., A philosophy of war (2007). Recuperado de

https://books.google.es/books?hl=es\&lr=\&id=0VezprbDgFoC\&oi=fnd\&pg=PR5\&dq=Philosophy+war\&ots=GFi8C9TBqg\&sig=G5 MTIn4lMHqHERa0mULJBV09QJo (Accedido 15/05/2020)

${ }^{53}$ BOOKCHIN, M., Remaking society (Nueva York 1989); ID., La ecología de la libertad (Madrid 1999); KROPOTKIN, P. A., DÍAZ, C., La moral anarquista (Barcelona 1978).

${ }^{54}$ WALLERSTEIN, I., BALIBAR, E., Raza, nación y clase (Barcelona 1991).

${ }^{55}$ El Protocolo I restringe las armas no detectables de fragmentación, el Protocolo II restringe las minas terrestres y las armas trampa, el Protocolo III restringe las armas incendiarias, el Protocolo IV restringe las armas láser cegadoras, el Protocolo V establece obligaciones y mejores prácticas para la limpieza de los restos explosivos de la guerra.
} 
una realidad determinada por dinámicas de explotación. Donde el animal “arma”, recorre todos los recovecos de nuestra historia bélica, desde el Imperio Romano al Vietcong. Y cuya consideración en la historia bélica se ha limitado a ser simplemente un medio, y nunca un fin en sí mismos. Siendo precisamente esta consideración histórica sobre el uso en el conflicto, la clave que debe amparar una regulación internacional vinculante sobre los usos de los animales en conflicto bélico, que supere los marcos dialécticos del "héroe animal", sustituyéndolo por el de animal "víctima" de las dinámicas de opresión que se aceleran y profundizan en dinámicas de conflicto armado.

Y es que, aunque la conversión de animales en "combatientes" aparentemente pueda presentarse como una conquista por lo que respecta a la consideración jurídica del animal como sujeto activo de derecho. Este avance no supone, ni supondrá al largo plazo, una disminución de los abusos, maltrato y victimología de los mismos en el contexto del conflicto armado. De la misma forma que las convenciones de Ginebra o los DDHH no han podido acabar con prácticas de exterminio, abuso o tortura sobre humanos.

Y lo que es más importante, la consideración de animales "combatientes" tampoco profundizará en un reconocimiento de la "dignidad" del resto de especies. Ya que de hecho, esta codificación normativa, continuaría considerando como legítimo o éticamente aceptable el hecho de involucrar otras especies en los conflictos humanos. Unas especies, que continuarían participando para servir, única y exclusivamente a los propios intereses humanos, hecho que cristalizaría la dinámica de subyugación existente, consolidando el antropocentrismo como eje regulador de nuestras relaciones inter-especies.

La traslación de la consideración de combatiente a los animales utilizados en conflicto, pese otorgar estatus de sujeto jurídico activo al animal, lo haría únicamente al animal explotado en el conflicto, es decir aquel al cual se le reconoce la subyugación a una fuerza combatiente, y no a todos los animales, ni siquiera los de la misma especie del territorio en el que se produce al conflicto. Por tanto, esta solución no ofrece, "per $s e$ " una consideración del animal como sujeto activo de derechos por su mera existencia, sino por su participación activa dentro de una fuerza combatiente. Es decir, reconoce derechos al "animal-arma" de la misma manera que las provisiones relativas a las tropas combatientes degradan también la experiencia humana al supeditar dichos derechos precisamente a la participación activa en el conflicto.

Es, por tanto, necesario avanzar en una conceptualización post-humana de la seguridad, que sea capa de inferir relaciones basadas en la equidad, y las aportaciones de las especies al mantenimiento de un ecosistema que es clave para garantizar la propia vida sobre el planeta. Siendo imprescindible, establecer marcos reguladores claros, que prohíban expresamente el uso de animales en el conflicto armado, en tanto que especies ajenas a la naturaleza propia del mismo. Y así mismo se establezcan provisiones claras respecto a los impactos ecológicos de la actividad militar y pruebas de armamento, de tal forma que los animales, sean considerados "per se" como víctimas colaterales y sus derechos sean tenidos en cuenta en el ejercicio de la praxis militar. Una práctica, la de la guerra y el conflicto armado que tarde o temprano deberemos condenar a la extinción al no ser más que una muestra del horror y de la degradación sobre la vida.

Si bien, y debido a que un escenario de ausencia de guerras parece extremadamente difícil de conseguir bajo los marcos mentales actuales, que todavía visualizan las relaciones internacionales como si de un juego de suma cero se tratase, debemos erosionar en todo lo posible los medios mediante la cual esta se produce, sustrayendo todo apoyo basado en el reconocimiento de la dignidad del resto de especies. Dignidad que se ve afectada por la actividad bélica, una de las actividades más erosivas sobre el medio llevadas a cabo por nuestra especie.

\section{Referencias}

- BAUDRILLARD, J., The Gulf War did not take place (Indiana 1995)

- BBC., Afghan Taliban capture British military dog - BBC News (Londres 06/02/2014). Recuperado de https://www.bbc.com/news/world-asia-26062679 (Accedido 24/06/2020)

- BISHOP, H., Robbing the bees (Nueva York 2006)

- BLUMENTHAL, R. S. 2134 - 112th Congress (Washington 2011-2012): Canine Members of the Armed Forces Act (2012)

- BODANSKY, D., The art and craft of international environmental law (2010). Recuperado de https://books.google.es/books?hl=es\&lr=\&id=eVeuV1EGna8C\&oi=fnd\&pg=PR7\&dq=internation al+environmental+law\&ots=8EtBbmCzlc\&sig $=$ WFkDTHPuHl18bfTkzJoifamaEgQ (Accedido $14 / 06 / 2020)$

- BOOKCHIN, M., Remaking society (Nueva York 1989)

- BOOKCHIN, M., La ecología de la libertad (Madrid 1999)

- BOOKCHIN, M. Ecología y pensamiento revolucionario (Mallorca 2019, 1a ed.)

- BRAIDOTI, R., Lo Posthumano (Barcelona 2015, 1a ed.) 
- BURNS, J., Sarajevo Journal; In the Zoo's House of Horrors, One Pitiful Bear. NYTimes (16/10/1992). Recuperado de: https://www.nytimes.com/1992/10/16/world/sarajevo-journal-in-thezoo-s-house-of-horrors-one-pitiful-bear.html (Accedido 14/06/3030)

- CARTER, B., CHARLES, N., Animals, Agency and Resistance. Journal for the Theory of Social Behaviour 43/3 (2013) 322-340. https://doi.org/10.1111/jtsb.12019

- CHOMSKY, N. La quinta libertad: la intervención de los Estados Unidos en América Central y la lucha por la paz (Barcelona 1999, 1a ed.).

- CHOMSKY, N., On Western Terrorism: From Hiroshima to drone warfare (London 2015). Recuperado de http://ebookespacio.info/on-western-terrorism-from-hiroshima-to-drone-warfarepdf-es-descargar-noam-chomsky.pdf (Accedido 14/05/2020)

- DARACK, E., Weaponizing Weather: The Top Secret History of Weather Modification. Weatherwise 72/2 (2019) 24-31. https://doi.org/10.1080/00431672.2019.1559400

- DELANEY, L., Military apllications of apiculture: The (other) nature of war (2000). Recuperado de https://apps.dtic.mil/dtic/tr/fulltext/u2/a600636.pdf (Accedido 03/04/2020)

- DELEUZE, G., GUATTARI, F., Millepiani (Roma 2006)

- FELdBLUM, J. T., MANFREDI, S., GILBY, I. C., PUSEY, A. E., The timing and causes of a unique chimpanzee community fission preceding Gombe's "Four-Year War." American Journal of Physical Anthropology 166/3 (2018) 730-744. https://doi.org/10.1002/ajpa.23462

- FETTER, S., Ballistic missiles and weapons of mass destruction: What is the threat? What should be done? International Security Journal 1/16 (1991) 5-42

- FREUD, S., Beyond the Pleasure Principle. The Standard Edition of the Complete Psychological Works of Sigmund Freud, Volume XVIII (1920-1922): Beyond the Pleasure Principle, Group Psychology and Other Works (Londres, 1920) 1-64. https://doi.org/10.3366/pah.2015.0169

- GIBBENS, S., Why These Animals Were Accused of Being International Spies. National Geographic (Londres 2018). Recuperado de https://www.nationalgeographic.com/news/2018/02/lizard-nuclear-spying-animal-espionage-spd/ (Accedido 12706/2020)

- GOODALL, J., In the shadow of man (Nueva York 2000)

- HEDIGER, R., Animals and War: Studies of Europe and North America (Leiden/Boston 2013)

- ICJ., Legality of the Threat or Use of Nuclear Weapons, Advisory Opinion (1996). ICJ-Reports.

- IDF., OKETZ Unit (2020). Accedido 15/06/2020, de https://www.idf.il/en/minisites/oketz-unit/

- KANT, I., Fundamentación para una metafísica de las costumbres (Madrid 2002)

- IRIGARAY, L., Speculum. L'altra donna (Roma 2010)

- KEAN, H., Animals and war memorials: Different approaches to commemorating the human-animal relationship. In Animals and War (2013) 237-262. Brill. Recuperado de https://brill.com/view/book/edcoll/9789004241749/B9789004241749-s012.xml (Accedido $14 / 06 / 2020)$

- KEELEY, L. H., War before civilization (Boston 1996)

- KLEM, E., BAKRAČ, S., MILANOVIĆ, M., Ecological consequences of warfare. Vojno Delo, 69/4 (2017) 126-133. https://doi.org/10.5937/vojdelo1704126K

- KROPOTKIN, P. A., DÍAZ, C., La moral anarquista (Barcelona 1978)

- LONDONO, E., Military Dog Captured by Taliban Fighters. Washington Post (28/04/2014). Recuperado de https://www.washingtonpost.com/world/national-security/military-dog-capturedby-taliban-fighters-who-post-video-of-their-captive/2014/02/06/c8d0f8f0-8f44-11 e3-84e127626c5ef5fb_story.html (Accedido 15/06/2020)

- LORETZ, J., The animal victims of the Gulf War. Phisicians for Social Responsability (1991) $221-$ 225. Recuperado de https://www.ippnw.org/ ippnwor1/pdf/mgs/psr-1-4-loretz.pdf (Accedido $14 / 05 / 2020)$

- LUND, M. H., Prehuman genocide. Human Evolution, 10/3 (1995) 225-231. https://doi.org/10.1007/BF02438975

- MACGREGOR, M., The story of Rome (Londres 2006)

- MERO, T., The Martens Clause, principles of humanity, and dictates of public conscience. American Journal of International Law 94/1 (2000) 78-89. Recuperado de https://www.cambridge.org/core/journals/american-journal-of-international-law/article/martensclause-principles-of-humanity-and-dictates-of-publicconscience/F55EECE5BED3DDB9D78162DA4509A03A (Accedido 11/05/2020)

- MOSElEY, A., A philosophy of war (2007). Recuperado de 
https://books.google.es/books?hl=es\&lr=\&id=0VezprbDgFoC\&oi=fnd\&pg=PR5\&dq=Philosophy +war\&ots=GFi8C9TBqg\&sig=G5MTIn4lMHqHERa0mULJBV09QJo (Accedido 15/05/2020)

- NOWROT, K., Animals at War: The Status of "Animal Soldiers" under International Humanitarian Law on JSTOR. Historical Social Reserach 154/2 (2015) 128-150

- RODRÍGUEZ-ÁLVAREZ, J., Rethinking Paradigms in the Technolo-ecological Transition. Journal of Law 1/2 (2019) 169-184

- RODRÍGUEZ, J., La civilización ausente: Tecnología y sociedad en la era de la incertidumbre (Oviedo 2016, 1a ed.)

- SALAS, J., El cruel asesinato que desató la primera guerra entre primates no humanos de la historia | Materia (14/01/2014). Recuperado de http://esmateria.com/2014/01/14/el-cruel-asesinato-quedesato-la-primera-guerra-entre-primates-no-humanos-de-la-historia/ (Accedido 18/04/2020)

- SÁNCHEZ-VÁZQUEZ, M. J., Ética e infancia: el niño como sujeto moral. Fundamentos En Humanidades 15/1 (2007) 179-193

- SANDS, P., Principles of International Environmental Law (2012). Recuperado de https://books.google.es/books?hl=es\&lr=\&id=Cz8hAwAAQBAJ\&oi=fnd\&pg=PR5\&dq=internati onal+environmental+law\&ots=efBBbwBntt\&sig=N5MO47bxGX69hwZfX4HRXrJ5Y8Y (Accedido 04/06/2020)

- SANTAYANA, G., Soliloquies in England and lates soliloquies (Nueva York1933)

- SCHOPENHAUER., A., The World as Will and Representation. Philosophy and Phenomenological Research, Vol. 20 (London 1959). https://doi.org/2104368

- SHAPIRO, P., Moral agency in other animals. Theoretical Medicine and Bioethics 27/4 (2006) 357373. https://doi.org/10.1007/s11017-006-9010-0

- SIPRI., Ecological Consequences of the Second Indochina War (1976)

- UK, N. A., World War II (2020). Recuperado de https://www.nationalarchives.gov.uk/education/worldwar2/theatres-of-war/westerneurope/investigation/resistance/sources/docs/4b/ (13/05/2020)

- VANDERBLIT, T. The CIA's Most Highly-Trained Spies Weren't Even Human (Washington 2013)

- VEBLEN, T., The Theory of the Leisure Class. A Penn State Electronic Classics Series Publication (Oxford 2009)

- WALLERSTEIN, I., BALIBAR, E., Raza, nación y clase (Barcelona 1991)

- WHITE, H. (n.d). Mithridatic Wars. Recuperado de http://www.perseus.tufts.edu/hopper/text?doc=Perseus\%3Atext\%3A1999.01.0230\%3Atext\%3DM ith. (Accedido 04/06/2020)

- WHITE, L., Tecnología medieval y cambio social (Barcelona 1973) 